\title{
Do dogs rescue their owners from a stressful situation? A behavioral and physiological assessment
}

\author{
Fabricio Carballo ${ }^{1,2}$. Victoria Dzik ${ }^{1,3} \cdot$ Esteban Freidin $^{4} \cdot$ Juan Pablo Damián ${ }^{5} \cdot$ Emma B. Casanave $^{2}$. \\ Mariana Bentosela ${ }^{1,3}$
}

Received: 20 March 2019 / Revised: 12 December 2019 / Accepted: 21 December 2019 / Published online: 6 January 2020

○) Springer-Verlag GmbH Germany, part of Springer Nature 2020

\begin{abstract}
Rescue behavior is considered a type of pro-social response, defined as a voluntary action directed to benefit another individual who is in a stressful or dangerous situation. In two experiments, we investigated whether dogs would rescue their owners when the person was trapped inside a wooden box and emitted clear signs of stress. The performance of these dogs was compared against that of a control group in which the owners remained calm while trapped. In addition, to assess if training modulated this behavior, we tested a group of dogs from the military trained in search and rescue tasks (Experiment 1). Results showed that dogs opened the box more frequently when the owner pretended to be stressed than when calm. Training shortened latencies to open the door but not the frequency of the behavior. In Experiment 2, we investigated if emotional contagion could be a possible mechanism underlying dogs' rescue responses by measuring dogs' behavior, heart rate, and saliva cortisol level in the stressed and calm conditions, and also controlled for obedience by having the calm owners call their pets while trapped. We replicated the findings of Experiment 1 as more dogs opened the door in the stressed owner condition than in the calm condition. In addition, we observed an increase in heart rate across trials in the stressed condition and a decrease across trials in the calm condition, but no differences in cortisol levels or stress-related behaviors between conditions. In brief, we found evidence that approximately half of the dogs without previous training showed spontaneous rescue behaviors directed to their owners. Neither was this behavior motivated by obedience nor by the motivation to reestablish social contact with the owner. We conclude that emotional contagion is a plausible mechanism underlying dogs' rescue behavior in the present protocol.
\end{abstract}

Keywords Pro-sociality $\cdot$ Training $\cdot$ Rescue behavior $\cdot$ Stress $\cdot$ Cortisol $\cdot$ Heart rate $\cdot$ Dogs

Electronic supplementary material The online version of this article (https://doi.org/10.1007/s10071-019-01343-5) contains supplementary material, which is available to authorized users.

Fabricio Carballo

facarballo01@gmail.com

1 Grupo de Investigación del Comportamiento en Cánidos (ICOC), Consejo Nacional de Investigaciones Científicas y Técnicas, Universidad de Buenos Aires, Instituto de Investigaciones Médicas (IDIM), Buenos Aires, Argentina

2 Departamento de Biología Bioquímica y Farmacia, Instituto de Ciencias Biológicas y Biomédicas del Sur (INBIOSUR), Universidad Nacional del Sur (UNS)-Consejo Nacional de Investigaciones Científicas y Técnicas (CONICET), San Juan 670, Piso 1, 8000 Bahía Blanca, Argentina

\section{Introduction}

Pro-social behavior refers to voluntary actions that benefit other individuals (Marshall-Pescini et al. 2016). From an ultimate level of analysis, explanations of pro-sociality involve finding the conditions under which benefiting others

3 Facultad de Medicina, Universidad de Buenos Aires, Instituto de Investigaciones Médicas A. Lanari, Buenos Aires, Argentina

4 Instituto de Investigaciones Económicas y Sociales del Sur (IIESS), UNS-CONICET, Bahía Blanca, Argentina

5 Departamento de Biología Molecular y Celular, Facultad de Veterinaria, Universidad de La República, Montevideo, Uruguay 
have increased an individual's fitness (Bshary and Raihani 2017; Nowak 2006). In this sense, comparisons of different species in their levels and forms of pro-social behaviors promise to help determine the factors underlying the evolution of pro-sociality (Brosnan 2013). In turn, from a proximal perspective, the definition of pro-sociality encompasses at least two elements, one cognitive and the other motivational. First, to provide benefits to others, individuals may need to infer others' internal states, needs, and goals. Second, individuals have to be motivated to provide the appropriate aid (Warneken 2015). In the present experiments, we focused on investigating whether dogs would behave pro-socially towards their owners in the form of a rescue response, and in doing so, we tested and controlled for different proximal explanations of this behavior.

Many animals have been shown to display pro-social behaviors towards conspecifics in experimental paradigms involving the sharing of food (Carter et al. 2013; QuervelChaumette et al. 2015) and the provision of help (Plotnik et al. 2011). An outstanding example has been the experimental observation of rescue behavior of both ants and rats towards their group or cage mates, respectively, from a stressful situation (Bartal et al. 2011; Nowbahari et al. 2009). Though rats' behavior was interpreted as empathic and goal-directed (Bartal et al. 2011), a similar behavior in simpler organisms such as ants, as well as subsequent experimental controls, have challenged cognitively complex explanations of rescue behavior in nonhuman animals (Silberberg et al. 2013; Vasconselos et al. 2012). Still, it remains debated whether mammals other than primates show prosociality based on emphatic-like abilities (Marshall-Pescini et al. 2016).

We here argue that dogs (Canis familiaris) are suitable and interesting for the study of pro-sociality, and in particular, rescue behavior directed towards people. Dogs have been sharing the same ecological niche with humans for at least 15,000 years (Galibert et al. 2011; Vila et al. 1997) and presumably have evolved specific socio-cognitive skills that allowed them to adapt to human environments rich in interdependent affordances (Hare and Tomasello 2005; Miklósi and Topál 2013). Furthermore, they usually live in people's homes and are integrated in human societies with countless opportunities to learn from humans (Udell et al. 2008). In terms of communicative skills, dogs have been shown to be proficient at following human cues (e.g., pointing gestures; Kaminski and Nitzschner 2013), discriminating human generous and selfish attitudes both directly (Carballo et al. 2015; Kundey et al. 2010a; Nitzschner et al. 2012) and indirectly by watching third-party interactions (Freidin et al. 2013; Kundey et al. 2010b; Marshall-Pescini et al. 2011), and distinguishing some human emotions relying on compound visual and acoustic stimuli (Albuquerque et al. 2016;
Merola et al. 2013) as well as visual (Müller et al. 2015) and olfactory (D'Aniello et al. 2018) cues alone. In fact, the expression of these abilities develops with dogs' social experiences (Barrera et al. 2011; Carballo et al. 2017; D'Aniello et al. 2017). Importantly, dogs are able to form strong social bonds with people (Nagasawa et al. 2015; Prato-Previde et al. 2003). Attachment to humans could be especially relevant in the study of dogs' pro-social behavior towards people given that bonding has been shown to mediate pro-sociality in a variety of species (Freidin et al. 2017).

Hitherto, the available evidence regarding dogs' prosocial behavior directed towards people is scant and inconclusive. Whereas dogs have shown pro-social sharing of food with a familiar dog in a laboratory paradigm (Quervel-Chaumette et al. 2015), they did not share food with their owner in the same paradigm (Quervel-Chaumette et al. 2016). Probably, the use of food in this test was inappropriate, given that, in everyday interactions, food sharing is asymmetric between dogs and humans. Moreover, studies using prosocial tasks that did not involve food also yielded mixed results. In a task in which dogs had to inform their owners about the location of a hidden object, subjects displayed signaling behaviors (gaze alternation and orientation) mostly when the dogs themselves were interested in the object, but not when only their owners were looking for it (Kaminski et al. 2011). In addition, Macpherson and Roberts (2006) did not observe helping requests in dogs (attracting a stranger's attention) when using a protocol in which their owners simulated a heart attack or being trapped under a bookcase. Differently to Macpherson and Roberts' study, which assessed whether dogs sought third party human help, in the current study we focused on whether dogs would directly provide aid to their stressed owners.

In contrast to the aforementioned inconclusive results, Custance and Mayer (2012) compared dogs' behavior in a situation where the owner or a stranger pretended to cry, to a control condition in which the person hummed a song, and found that dogs were more likely to approach the person in the crying condition than in the control condition. In turn, Bräuer et al. (2013) found that, after training, dogs would press a button to open a door that allowed an unfamiliar person to reach a target object. The fact that the dog was unacquainted with the person makes this result particularly interesting, because familiarity is expected to increase helping. However, dogs' help (i.e., pressing the button) only occurred when the person was allowed to communicate spontaneously with the dog, that is naturally, as they would in an everyday situation, and when directly pointed at the button (Bräuer et al. 2013). These procedural facts do not rule out the possibility that dogs were simply following the person's command (pointing), rather than inferring the person's intention (Szetei et al. 2003). Furthermore, the fact that the action to 
press the button had been trained with food rewards left open the possibility that the animal performed the target action under the expectation of receiving food.

In conceptual terms, Nowbahari and Hollis (2010) defined rescue behavior as the provision of aid to a distressed individual at a cost and without immediate reward to the rescuer. Based on this definition, to our best knowledge, only a single study to date has directly addressed rescue behavior in dogs. Sanford and colleagues (2018) evaluated dogs' rescue behavior towards their owners in a trapped paradigm similar to that used with ants and rats (Bartal et al. 2011; Nowbahari et al. 2009). During the test, the owner was enclosed in a small room asking for help and pretending to cry (stressed group) or humming (control group). The dog could open a transparent Plexiglas door, locked with a magnet, to access the owner and end his/her stress. These authors did not find any significant difference in the number of openings or in the latency to open the door between conditions, and claimed that these inconclusive results could be the consequence of dogs opening the door unintentionally given that the door locking device opened too easily. Furthermore, the owners were not allowed to look at the dog or provide any gestural cue which could have made it difficult for dogs to understand the situation. However, when only the behavior of those dogs that opened the door was compared between conditions, animals in the stressed owner condition showed shorter latencies to open the door compared to dogs in the control group. Moreover, subjects' stress responses (vocalizations, panting, shaking off, yawning, and scratching) were reduced in dogs that opened the door and increased in those that did not, compared to a baseline. Although Sanford and colleagues (2018) highlighted that dogs could attend to their owners' emotional state, they could not find reliable evidence of rescue behavior. Nonetheless, it remains untested whether dogs would spontaneously help their owners if the person made a direct request for it. It is also important to notice that half of the dogs in Sanford et al. (2018) study were certified trained working dogs. Given that specific training has been shown to modulate the behavioral reaction to separation in working dogs (Fallani et al. 2007), the fact that some dogs were trained limits the capacity of the study to clarify the proximal mechanisms underlying rescue behavior in dogs and to generalize the obtained results to untrained dogs.

The aim of the present study was to evaluate whether dogs would exhibit rescue behavior directed towards their owners in a similar paradigm to that used by Sanford et al. (2018), and to test for alternative proximal mechanisms that may underlie this behavior. For this purpose, we enclosed the dog's owner in a wooden box with a transparent Plexiglas door. To release the person, the dog had to remove a stone that kept the door closed or pull the door with its nose or paw. We compared dogs' performance when the owner emitted clear signs of stress (shouted for help) against the performance of dogs whose owners remained calm inside the box. This calm condition was aimed at controlling for the possibility that seeking social contact motivated door openings. In Experiment 1, the owner in the calm condition emitted no vocalizations. In contrast, in Experiment 2, the owner in the calm condition called the dog in a calm manner to control for the possibility that dogs in the stressed condition of Experiment 1 and 2 opened the door following their owners' command. Unlike Sanford et al. (2018), we allowed the owner to emit spontaneous signs of stress in the stressed owner conditions (see more details about the experimental instructions for owners in the Materials and Methods section).

In terms of the alternative proximal mechanisms assessed, we here focused on evaluating training experience (comparing trained military dogs against pets in Experiment 1), obedience (controlled for in Experiment 2 by assessing the dog's responses to the owner's calls in the calm condition), and emotional contagion (indirectly evaluated in Experiment 2 with behavioral and physiological measures).

Training could be a relevant variable given that it increases the capacity of dogs to collaborate with people during specific tasks (Jezierski et al. 2014; Fallani et al. 2007; Wallis et al. 2015). In Experiment 1, in addition to untrained family dogs, we tested military dogs trained in search and rescue tasks, which were nonetheless unfamiliar with the testing procedure and apparatus. The comparison between trained and untrained dogs was used to assess the role of structured experience in the display of dogs' prosocial behaviors and was also meant to provide information regarding the generalizability of trained abilities to novel tasks.

Additionally, it is possible that dogs learn during their ontogeny that obedience to commands, such as responding to a call, is rewarded by their owners (Schwab and Huber 2006). For this reason, in Experiment 2, we controlled for the possibility that dogs simply opened the door as a response to their owners' calling their names.

Last, we tested for an affective mechanism. Empathylike responses have attracted much attention as a possible mechanism underlying rescue behavior. For rats in the trapped-mate paradigm, Bartal et al. (2009) interpreted rescue behavior as motivated by emphatic concern, whereas Silberberg et al.'s (2013) results were more consistent with the possibility of rats acting to regain social contact with their mates. In the case of dogs, the only existing evidence to date shows that subjects showed increased stress responses when they were unable to rescue their owners, whereas stress responses decreased when they succeeded (Sanford et al. 2018). This points towards the possibility that dogs' rescue behavior is related to basic forms of empathy such as emotional contagion (i.e., experiencing similar affective states as those observed in a target individual). In contrast 
with the notion of empathic concern, emotional contagion does not require individual's recognition of others' mental/ affective states (Hatfield et al. 1994; Panksepp and Panksepp 2013). Indeed, previous research indicates that dogs can show emotional contagion from their owners (Huber et al. 2017; Sümegi et al. 2014; Yong and Ruffman 2014), and emotional contagion has been proposed as an explanation for dogs' comforting behavior (Custance and Mayer 2012). To better understand the role of emotional contagion in rescue behavior, we conducted a second experiment, in which we recorded both behavioral and physiological measures of dogs' stress (saliva cortisol and heart rate), and compared these measures between the stressed owner and the calm owner conditions. Both heart rate and saliva cortisol concentration have been used as indicators of dog's stress and emotional states in experimental tasks before (e.g., Barber et al. 2017; Beerda et al. 1997, Beerda et al. 1998; King et al. 2003; McGowan et al. 2018; Palestrini et al. 2005).

\section{Ethical statement}

The protocol that involved the evaluation of the dogs and their owners was approved by the Institutional commission for the care and use of laboratory animals (CICUAL) at the Medical Research Institute, IDIM UBA-CONICET (Res. Nro. 040-16, 074-17), and complied with the current Argentine law of animal protection (Law 14.346). All owners involved were adults ( $>18$ years old) and expressed their consent for the participation of their dogs in the present studies.

\section{Experiment 1}

\section{Materials and methods}

\section{Subjects}

We recruited 47 pet dogs. Nine dogs had to be removed from the study because their owners did not follow the experimenters' instructions (i.e., they did not show signs of stress in the stressed owner condition or attempted to call dogs' attention in the calm owner condition). The final sample comprised 38 dogs that lived with their owners as pets since at least one year, were in good health, were between 6 months and 10 years old, and, according to the owner's report, had no formal obedience training. These subjects were randomly assigned to the stressed owner condition or the calm owner condition. The stressed owner condition comprised 22 dogs ( 8 males, 14 females; mean age in years $\pm 1 \mathrm{SD}: 4.3 \pm 2.29$ ) and their owners ( 6 men and 16 women); and the calm owner condition comprised $16 \mathrm{dogs}$
( 8 males, 8 females; mean age in years: $4.19 \pm 2.61)$ and their owners ( 8 men and 8 women).

In the condition with trained dogs, we had 10 military dogs that had been trained in search and rescue tasks at the Base Naval Puerto Belgrano, Argentina. Trained dogs were between 8 months and 10 years old ( 7 males, 3 females; mean age in years: $3.59 \pm 2.9$ ) and lived in the military base. The trainers were all men. These dogs received specific training sessions twice a day with the same trainer every day and were highly socialized since puppies. Thus, each dog in this group had a close relationship with one specific trainer (hereafter called "owner" for simplicity) and had daily contact with him. Furthermore, although these dogs lived in kennels at the military base, they also spent some weekends at their owners' home. Training tasks involved searching for missing persons or specific objects in an open field with obstacles. When dogs found the target they were taught to signal the position of the target by barking and standing in the correct location.

The sample comprised dogs of different breeds (see supplementary material, Table S1, for details).

\section{Apparatus}

To trap the owners, we used a manufactured wooden box of $1 \mathrm{~m}^{2}$ base and $1.75 \mathrm{~m}$ height, with a transparent Plexiglas' door (see Fig. 1), which allowed the dog to see the person inside. An elastic rope pulled the door to an open position. When closed, a $1.5 \mathrm{~kg}$ stone prevented the door from automatically opening, and dogs could open the door simply by removing the stone or putting their nose/paw in the gap between the door and the lateral wall of the box to pull the door. Tests were conducted at the owner's house for pet dogs and in a $10 \times 5 \mathrm{~m}$ room familiar to subjects inside the Army facilities for trained dogs.

\section{Procedure}

In sessions with family dogs, upon arrival at the house, the experimenter assembled the box (15 min), placed two video cameras in the room (SONYDCR-SR88 and SONY DCR 308 ), and explained the procedure to the owner (which varied depending on the condition, stressed or calm). During this period, the dog was allowed to explore the room and the box to habituate to the situation. Once built, the box remained opened so the dog could get inside during the habituation period. Then, the experimenter led the dog to an adjacent room, the owner got inside the box, and the experimenter put the stone that prevented the door from opening.

Once the owner was inside the box with the door closed, the experimenter left the room and allowed the dog to enter, which marked the beginning of the first trial. The behavior of the owner inside the box depended on the condition 


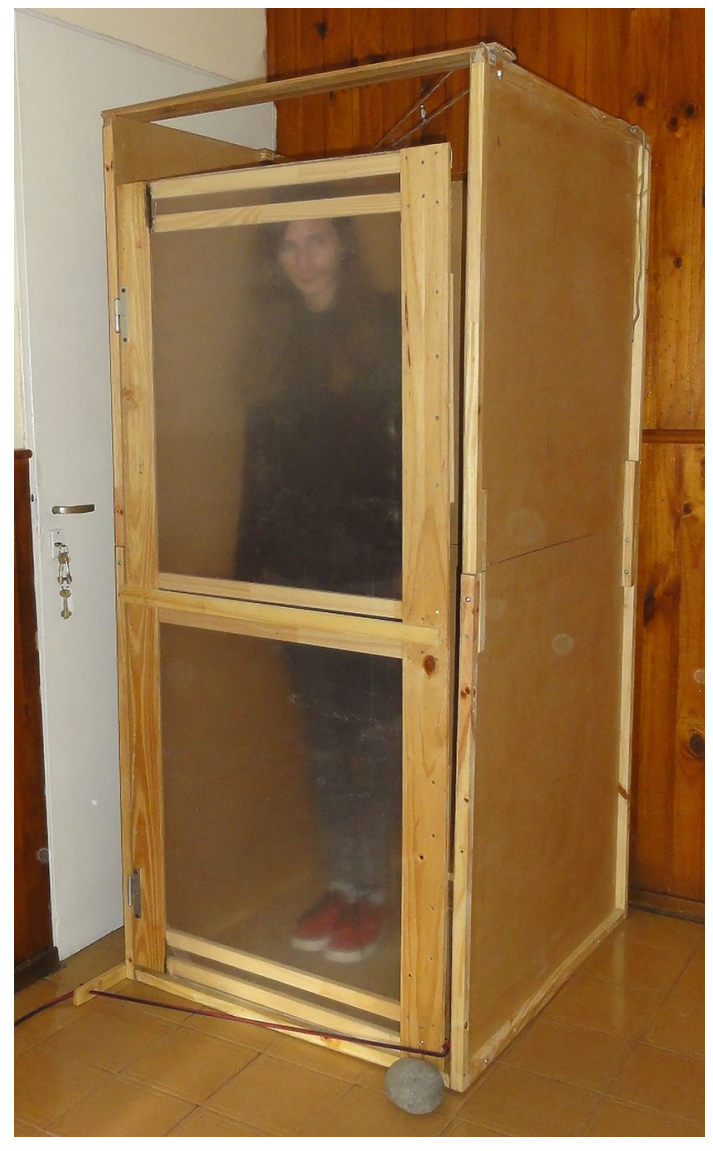

Fig. 1 Photo of the experimental apparatus with the owner inside. The owner expressed his consent to appear in this publication

(See Supporting information Video 1 with an example of an evaluated subject).

Untrained Dog with Stressed Owner (US): immediately after the dog entered the room the owner began crying for help, pretending to be locked inside the box and unable to leave. Owners were instructed to act as if stressed by the situation (i.e., to scream, pretend to cry, hit the walls of the box). To ensure understanding, we showed the owners a sample video on a smart phone of an experimenter performing the desired behavior. During trials, owners were told that they could call the dog by its name or do whatever they would do in a dangerous situation.

Untrained Dog with Calm Owner (UC): in this condition, the owner remained seated inside the box reading a book or using her/his cellphone, and was asked not to pay any attention to the dog or call the dogs' attention. In contrast with the US condition, owners did not show signs of stress in the UC condition, which was implemented to control for the possibility that the dog may open the box with the sole purpose of reestablishing contact with its owner.

Trained Dog with Stressed Owner (TS): the procedure in this condition was exactly the same as that used in the
US condition with the following differences: (1) the box was placed in a $10 \times 5 \mathrm{~m}$ room familiar for all subjects, and (2) before the first trial, the dog was allowed to explore the room and familiarize with the box for approximately $5 \mathrm{~min}$.

We ran a between-subject design in which each dogowner pair participated in only one condition. Each session comprised three trials, and each trial lasted until the dog opened the door or for a maximum of $120 \mathrm{~s}$. If the dog opened the door before $120 \mathrm{~s}$ elapsed, the owner left the box and verbally congratulated the dog. If, after $120 \mathrm{~s}$, the dog did not open the door, the experimenter re-entered the room, removed the dog from the room, and let the owner out of the box. During this period, the dog remained in an adjacent room with the experimenter and apart from its owner. After a 60 -sec break, the next trial began.

All trials were videotaped, and all measures were obtained from the videos.

\section{Data coding}

In each trial, we measured whether dogs opened the door, and the latency to open it defined as the time elapsed since the beginning of the trial until the dog opened the box or the trial ended, whichever occurred first. In trials were dogs did not open the door, we coded a maximum latency of $120 \mathrm{~s}$. We also scored the duration of the following behaviors in sec for each trial: (1) interaction with the box: time dogs spent in physical contact with the box (touching it with the mouth, paw, jumping on it, etc.); (2) proximity to the box: time dogs spent within $50 \mathrm{~cm}$ from the box; (3) proximity to the exit: time dogs spent within $100 \mathrm{~cm}$ from the door leading to the adjacent room where the experimenters were; (4) ears down: time dogs spent with the ears down; and (5) tail down: time dogs spent with the tail held lower than the plane of the back. Given that not all the trials had the same duration, we divided the duration of the aforementioned behaviors by the total duration of the respective trial. Thus, for the analysis, we used the proportion of time dogs spent performing each behavior by trial. We also coded the number of vocalizations (barks and whining) and lip licking when occurred. We divided the number of vocalizations and lip lickings by the duration of the trial to obtain a frequency by second as measure (frequency). Tail and ears down, lip licking, and vocalizations were interpreted as stress-related behaviors (Beerda et al. 1997).

Behavioral data were measured by one of the authors (VD) from the videos, and it is important to note that this person was blind to the hypotheses of the study. To assess reliability, 20 per cent of the trials were also coded by another author (FC). Inter-observer reliability was high (the inter-observer correlations of the different measures ranged from 0.84 to 0.99 , all $p$-values $<0.001$ ). 


\section{Data analysis}

Whether subjects opened the box door at least once (variable "open") was coded in a binary manner (0/1), and pair-wise comparisons between conditions were analyzed using Fisher's exact test. To control for sex, age, neutered state, and owner's sex, we also did Probit regression models of the variable open with condition US as intercept and the remaining conditions as predictors. Additionally, we counted the number of times each subject opened the door in the 3 trials (variable "total open", 0-3), and we compared conditions on this variable with Mann-Whitney $U$ tests. Last, to control for sex, age, neutered state, and owner's sex, we also did Tobit regression models of the variable total open (truncated at 0 and 3 ) with condition US as intercept and the remaining conditions as predictors. To analyze if the proportion of dogs that opened the door varied across trials, we used Cochran's test.

To analyze latencies, we ran a GLMM with condition as fixed factor, trial as a repeated measure, and the condition $\times$ trial interaction. Given that latencies were not normally distributed and the distribution was skewed towards higher values, we used a gamma probability distribution with $\log$ as linking function.

To evaluate differences among conditions in the duration or frequency of each behavior (interaction with the box, proximity to the box, proximity to the exit, ears down, tail down, vocalizations, and lip lickings), we used Mann-Whitney $U$ tests for pair-wise comparisons between conditions. Dogs with floppy ears (3 dogs in the UC condition) or no tail (1 in the US condition) were discarded from the analysis of measures concerning those body parts. For behaviors measured as durations, we used the mean of the proportion of time dogs spent performing the behavior of interest in the three trials. For behaviors measured as frequencies, we used the mean of the number (count) of instances of the behavior of interest in the three trials divided by the duration of that trial. For behaviors with low frequency of occurrence or duration, we present descriptive statistics (see Table S2 in supplementary materials) but do not report any inferential analyses.

\section{Results and discussion}

\section{Door opening}

Table 1 shows the proportion of dogs that opened the door by trial for each condition. The main comparisons of interest were those between US and UC conditions, and US and TS conditions for variables open and total open. Whereas 13 of $22 \operatorname{dogs}(59 \%)$ opened the box door at least once in the US condition, 3 out of 16 subjects (19\%) did so in the UC condition (Fisher's exact test, two tailed, $p=0.02$ ). In turn, 8 of the $10(80 \%)$ dogs opened the door in the TS condition, which did not differ from the performance in the US condition $(p=0.42)$. A Probit regression showed an effect of the UC condition (coefficient \pm standard error: $\beta=-1.12 \pm 0.45 p=0.01)$, and a non-significant effect of the TS condition $(\beta=0.61 \pm 0.53, p=0.24)$, both relative to the US condition as intercept $(\beta=0.23 \pm 0.27)$. These results remained qualitatively similar when we introduced dog's sex, age, neutered state, and owner's sex as controls in the regression (UC effect, $\beta=-1.53 \pm 0.54, p<0.01$; TS effect, $\beta=-0.49 \pm 0.80, p=0.54)$.

In terms of the variable total open, we observed a difference between US and UC conditions $(U=96, Z=2.36$, $p=0.02$ ). No differences were found between US and TS conditions $(\mathrm{U}=91, Z=-0.85, p=0.43)$. Tobit regressions confirmed these findings, even after controlling for dog's sex, age, neutered state, and owner's sex (UC, $\beta=-10.85 \pm 4.99$, $p=0.036$; TS, $\beta=-4.39 \pm 4.99, p=0.38$; intercept: US, $\beta=1.11 \pm 5.88, p=0.85$ ).

There were no significant differences in the proportion of dogs that opened the door across trials in any of the conditions (all $p$-values $>0.2$ ).

Summing up, present results indicate that dogs did more openings when their owners acted as if stressed than when they were in a calm state (Fig. 2).

\section{Latencies}

Table 2 shows the mean \pm 1 standard error (SE) for latencies to open the door in each trial and in each condition.

In terms of latencies to open the door, we found main effects of condition $[F(2,135)=7.55, p=0.001]$ and trial $[F(2,135)=12.54, p<0.001]$, and a tendency towards significance for the condition $\mathrm{x}$ trial interaction $[F(4,135)=2.38$,
Table 1 Proportion of dogs that opened the door by trial in each condition

\begin{tabular}{llll}
\hline Condition & Trial 1 & Trial 2 & Trial 3 \\
\hline Untrained dog with stressed owner & $0.45(10 / 22)$ & $0.54(12 / 22)$ & $0.50(11 / 22)$ \\
Untrained dog with calm owner & $0.06(1 / 16)$ & $0.12(2 / 16)$ & $0.18(3 / 16)$ \\
Trained dog with stressed owner & $0.60(6 / 10)$ & $0.80(8 / 10)$ & $0.70(7 / 10)$ \\
\hline
\end{tabular}




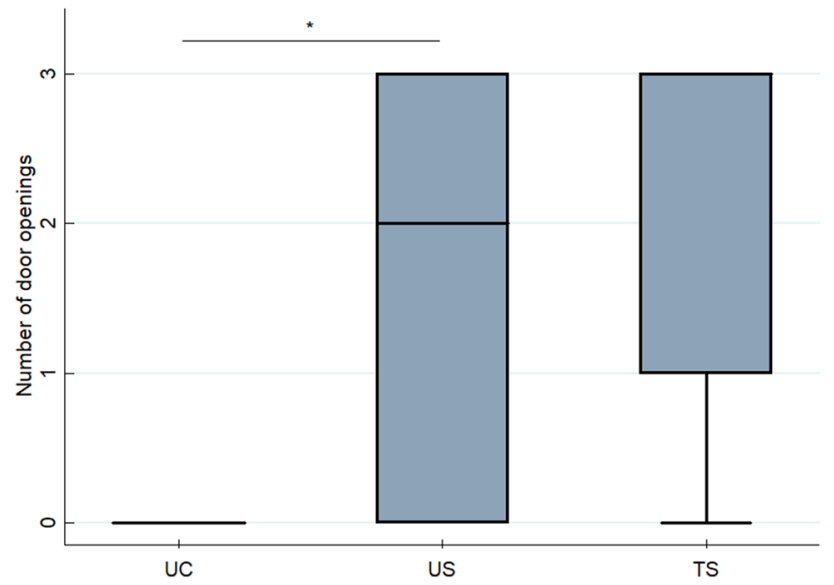

Fig. 2 Median number of door openings as a function of condition in Experiment 1. Error bars denote the minimum and maximum values. $U C$ Untrained dog with a calm owner, $U S$ untrained dog with a stressed owner, and TS trained dog with a stressed owner. * $p<0.05$

$p=0.054]$. Pair-wise comparisons showed that latencies in the US condition were shorter than in the UC condition [US vs UC: $t(135)=-2.09, p=0.03$ ]. In addition, dogs showed shorter latency to open the door in the TS than in the US condition $[t(135)=1.99, p=0.04]$. The fact that trained dogs opened the door with shorter latency than untrained dogs suggests that the former were more proficient at solving the task.

Dogs solved the task faster in the second and third trials compared to the first trial [Trial 1 vs Trial 2: $t(135)=3.52$, $p=0.001$; Trial 1 vs. Trial 3: $t(135)=2.89, p<0.01]$. There were no differences in latencies between the second and third trials $[t(135)=-1.38, p=0.01]$. Doing the analysis separately for each condition, we found that the reduction in the latency to open the door was significant in the TS condition [Trial 1 vs Trial 2: $t(135)=2.24, p=0.02$ ] and there was a tendency towards a significant reduction across trials in the US condition [Trial 1 vs Trial 2: $t(135)=1.96, p=0.051$ ] No other significant differences were found $(p>0.05)$.

\section{Other behavioral measures}

Table S2 in supplementary materials shows the descriptive data of all behavioral variables as a function of trial.

\section{Proportion of time performing each behavior}

We found differences among conditions only in the proportion of time dogs spent in proximity to the exit (Kruskal-Wallis: $H=5.92, p=0.04, N=48$ ). More specifically, dogs spent more time near the exit in the UC condition (Mean and SD: $25.95 \pm 32.76 \mathrm{~s}$ ) than in the TS condition (Mean and SD:2.04 $\pm 4.32 \mathrm{~s} ; Z=-2.22, p=0.02$ ), whereas there were no differences between US (Mean and SD: $15.47 \pm 30.58)$ and the $\mathrm{UC}$ conditions $(Z=-1.28, p=0.21)$ or between the US and TS conditions $(Z=-0.31, p=0.81)$. No other significant differences were found in any of the remaining behaviors (all $p$-values $>0.05$ ).

\section{Vocalization and lip licking}

There were no significant differences among conditions in the number of vocalizations or lip lickings (all $p$-values $>0.05$ ).

In conclusion, present results indicate that dogs did more openings when their owners acted as if stressed (US and TS conditions) than when they were in a calm state (UC). However, it is not clear whether dogs opened the door because of their owners' stress or whether such behavior has a different explanation. For instance, owners in the stressed conditions could call dogs by their names, whereas that was not allowed in the calm condition. Therefore, the differential door opening behavior found between stressed and calm conditions could be simply explained as a consequence of obedience to the owners' command in the former conditions. That is, dogs might have approached and interacted with the box after the owners called their names in the stressed conditions, leading to an increased probability of opening the door of the box. In Experiment 2, we controlled for the obedience explanation.

\section{Experiment 2}

In Experiment 1, dogs displayed responses effective at opening the box where their owners were trapped and stressed. We hypothesize that emotional contagion from the owner could be the mechanism underlying dogs' responses. According to this notion, dogs were more effective at opening the box in the stressed conditions than in the calm condition because they might have been more aroused and active in the former than
Table 2 Mean \pm 1 SE of the latency in seconds to open the door in each trial in each condition of Experiment 1

\begin{tabular}{lccc}
\hline Condition & Trial & \\
\cline { 2 - 4 } & 1 & 2 & 3 \\
\hline Untrained dog with stressed owner & $74.49 \pm 9.96$ & $74.49 \pm 9.96$ & $67.37 \pm 11.73$ \\
Untrained dog with calm owner & $119.50 \pm 0.50$ & $107.09 \pm 8.84$ & $102.14 \pm 9.67$ \\
Trained dog with stressed owner & $64.85 \pm 17.08$ & $29.79 \pm 15.10$ & $45.27 \pm 16.81$ \\
\hline
\end{tabular}


the latter, after their owners' differential affective responses. To explore if dogs become stressed when their owners show signs of stress in the rescue paradigm, we took both physiological and behavioral measures of dogs' stress in Experiment 2. In addition, we controlled for the possible effect of obedience to the owner's command; hence, the owner called the dog by its name in both calm and stressed conditions in Experiment 2.

\section{Subjects}

We recruited 48 pet dogs with the same selection criteria used in Experiment 1. Five dogs had to be discarded from the sample due to technical problems during physiological data collection or because their owners did not follow the experimenters' instructions (i.e., they did not show signs of stress in the stressed owner condition, or US, or did not call the dog in the calm call condition, or UCC). The final sample comprised 43 dogs that were between 1 and 11 years old. The stressed owner condition comprised 20 dogs ( 12 males and 8 females; mean age in years \pm 1 SD: $5.46 \pm 2.58)$ and their owners ( 7 men and 13 women); and the Calm Call condition comprised 23 dogs (10 males and 13 females; mean age in years: $5.27 \pm 3.07$ ) and their owners (23 women), (See Table S3 in supplementary materials for more details).

\section{Apparatus}

We used the same apparatus as in Experiment 1, but, unlike Experiment 1, testing was conducted in an outdoor arena $(10 \times 15 \mathrm{~m})$ belonging to the Argentine National Research Council (CONICET) in Bahia Blanca. Next to this arena, there was a building with an indoor waiting room where saliva samples and heart rate measures were collected (See procedure). This change of location relative to the previous experiment (in which tests were conducted at dogs' homes) implied that owners had to bring their pets to the testing location in Experiment 2.

\section{Procedure}

When the dogs arrived at the experimental location, they were allowed to explore both the indoor and outdoor environments for $10 \mathrm{~min}$. We took this time to instruct the owners on how they had to behave during trials. Then, the owner called the dog to the waiting room and the experimenters proceeded with the initial physiological measurements.

\section{Physiological measurements}

\section{Saliva sampling and cortisol determination}

Dogs were paired by condition at the time of the testing, so a dog from the US condition was always followed by one from the UCC one. Therefore, dogs from both conditions were tested at similar times in the day thus controlling for variations in cortisol levels across the day. Saliva samples were collected immediately before and 20 min after the beginning of the first trial. The procedure used to collect the saliva samples was according to Damián et al. (2018). We used cotton rolls (CROSSTEX N ${ }^{\circ} 2$ MEDIUM, USA) soaked with citric acid (5\%) and then dried in an oven at $60{ }^{\circ} \mathrm{C}$ for $5 \mathrm{~h}$. Citric acid was used to increase the flow of saliva (Beerda et al. 1998; Damián et al. 2018; Walker et al. 2016). Each roll of cotton was gently placed in the dog's mouth for $1 \mathrm{~min}$ and, immediately after, the cotton was put in a $15 \mathrm{~mL}$ falcon tube (with a nylon mesh of $2 \mathrm{~mm}$ diameter pores) which was placed on ice. Samples were collected in duplicate each time. The tubes were centrifuged at $3500 \mathrm{rpm}$ for $15 \mathrm{~min}$ at $4{ }^{\circ} \mathrm{C}$, and saliva samples were stored at $-20{ }^{\circ} \mathrm{C}$. Cortisol saliva concentrations were determined by a competitive chemiluminescent enzyme immunoassay (Immulite ${ }^{\circledR} 1000$ analyzer using a Siemens Cortisol kit; Los Angeles, USA).

\section{Heart rate (HR) response}

HR was monitored by both manual and electronic methods. The manual HR measure was recorded using a stethoscope (pediatric double bell, CORONET HS-30H-Pediatric), and the electronic HR measure was obtained through a continuous recording device (Polar H10 heart rate sensor, Finland). The manual recording of dog's cardiac response was collected via auscultation for $30 \mathrm{~s}$ before the first trial and immediately after the third (last) trial in the indoor waiting room with two experimenters and the owner present. For the automatic recording, we first applied a conductive gel over the dog chest, then attached the elastic band with sensor electrodes of the Polar device to the dog, and finally obtained electronic HR data using a Polar beat app in a smart phone. Each HR Polar measure involved the recording of $120 \mathrm{~s}$ of HR activity. After 3 min of habituation to the Polar device, we took a baseline measure before the first trial (POLAR_BL), then a measure in each trial (POLAR_T1, POLAR_T2, and POLAR_T3), and one last measure after the last trial (POLAR_POST).

\section{Test}

Immediately after the POLAR_BL measure, the dog waited in the indoor waiting room, while one experimenter led the owner to the outdoor testing arena and locked him/her inside the box. Then, the dog was released, which marked the begining of the first trial. Depending on the assigned condition, the owner was instructed to behave as follows:

Untrained Dog with Stressed Owner (US): this condition was similar to the US condition described in Experiment 1; this time, though, the frequency of each owner's 
call to his/her dog (i.e., the owner calling the dog by its name) was recorded in each trial for reasons that we explain next.

Untrained Dog with Calm Calling Owner (UCC): we here used a similar procedure to that described for the UC condition in Experiment 1. However, this time, each dogowner dyad was yoked with a dyad from the US condition so that both conditions were matched in terms of the frequency of owner's calls. This was done to control for the possibility that dogs opened the box out of obedience after their owners call their names, and not driven by their owners' affective state. To achieve this yoking, each owner in the UCC condition was instructed on which moment and how many calls to make in each trial to match the number of calls of the corresponding owner in the US condition. For this, the owner had a stopwatch and a table that specifed the time in the trial for each of his or her calls. In contrast to the US condition, though, owners were asked to call their dogs in a neutral tone of voice and do not establish eye contact with the dog in the UCC condition.

Similar to Experiment 1, each dog-owner pair received three 120 -sec trials.

\section{Data coding and analyses}

For all behaviors, we did the same coding and analyses than in the previous experiment. For the ears down measure, we had to remove dogs with floppy ears (1 in the UCC condition), and for the tail down measure, we had to remove dogs without tail ( 1 in the UCC condition, and 5 in the US condition).

Cortisol measures were analyzed with a mixed-model ANOVA using the mixed model of SAS (SAS University Edition). The model included condition as a between-subject factor (US vs. UCC), time as within-subject factor (before the first trial vs. after the last trial), the condition $\times$ time interaction, and individual dogs as a random effect. We used paired $t$-tests to compare HR measured by auscultation before the first trial (Pre) and after the last trial (Post) both between (US vs. UCC) and within conditions (Pre vs. Post), given that these measures were normally distributed according to Shapiro-Wilks test. For HR measures obtained with the POLAR device, we ran a GLMM with condition (US vs. UCC) and phase (baseline [BL], experimental [EXP], and POST) as fixed effects, trial as nested factor within the EXP phase, and individual subjects as a random factor. We also analyzed the condition $\times$ phase interaction and the condition $\times$ trial (within phase) interaction to assess if HR varied across trials in the experimental phase differently between conditions. Results were considered significant with $\alpha=0.05$.

\section{Results and disussion}

\section{Door opening}

Table 3 shows the proportion of dogs that opened the door by trial in each condition. Whereas 13 of $20 \operatorname{dogs}(65 \%)$ opened the box door at least once in the US condition, 8 out of 23 subjects ( $35 \%$ ) did so in the UCC condition (Fisher's exact test, two tailed, $p=0.07)$. A Probit regression showed an effect of the UCC condition $(\beta=-0.78 \pm 0.39, p=0.049$, relative to the US condition as intercept, $\beta=0.39 \pm 0.27$ ), and these results remained qualitatively similar when we introduced dog's sex, age, neutered state, and owner's sex as controls in the regression $(\beta=-1.08 \pm 0.45, p=0.017)$.

In terms of the variable total open, a Mann-Whitney $U$ test showed that dogs in the US group opened the door more times than dogs in the UCC group $(U=117, Z=2.75$, $p=0.005$ see Fig. 3). Tobit regressions confirmed these findings, even after controlling for dog's sex, age, neutered state, and owner's sex $(\beta=6.29 \pm 2.63, p=0.022)$.

There were no trial differences in the proportion of dogs that opened the door in any condition (all $p$-values $>0.05$ ) (Fig. 3).

\section{Latencies}

We found a main effect of condition $[F(1,118)=10.35$, $p<0.01]$ and a tendency towards significance in the effect of trial $(F(2,118)=2.74, p=0.069)$, but there was no significant condition $\times$ trial interaction $[F(2,118)=0.54$, $p=0.58]$. Dogs had shorter latencies to open the box in the US condition than in the UCC condition $[t(118)=-2.97$, $p<0.01]$. Subjects opened the door faster in the third trial than the first trial $[t(118)=2.08, p=0.03]$. Analyzing each group separately, we observed that the latency to open the door tended to decrease from the first trial to the second trial $[t(118)=1.92, p=0.057]$ and from the first trial to the third trial $[t(118)=1.95, p=0.053]$ in the US condition, but not in the UCC condition (all $p$-values $>0.3$ - see Table S4 in supplementary materials).

Table 3 Proportion of dogs that opened the door by trial in each condition

\begin{tabular}{llll}
\hline Condition & Trial 1 & Trial 2 & Trial 3 \\
\hline $\begin{array}{l}\text { Untrained dog } \\
\text { with stressed } \\
\text { owner }\end{array}$ & $0.65(13 / 20)$ & $0.66(12 / 18)$ & $0.52(10 / 19)$ \\
$\begin{array}{l}\text { Untrained dog } \\
\text { with calm call- } \\
\text { ing owner }\end{array}$ & $0.13(3 / 22)$ & $0.26(6 / 23)$ & $0.18(4 / 22)$ \\
\hline
\end{tabular}




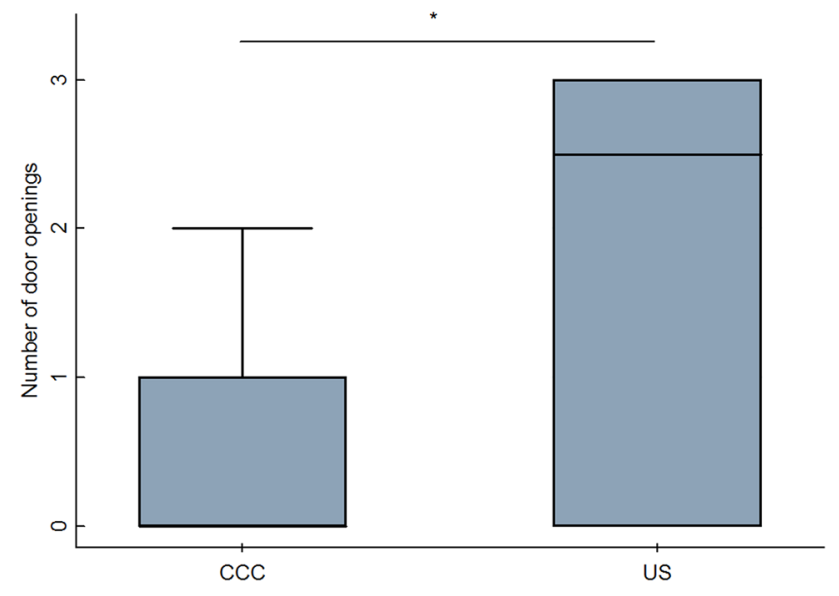

Fig. 3 Median number of door openings as a function of condition in Experiment 2. Error bars denote minimum and maximum values. $U C C$ Untrained dog with calm calling; and $U S$ untrained dog with stressed owner. $* p<0.05$

\section{Other behavioral measures}

Descriptive data of other behaviors are presented in Table S4 in supplementary materials.

\section{Proportion of time performing each behavior}

We found significant differences between conditions in the mean proportion of time dogs spent in contact with the box $(Z=-2.41, p=0.01, n=43)$ and in proximity to the exit $(Z=-2.22, p=0.02, n=43)$. Dogs in the US condition spent more time near the box (mean \pm 1 SD: $0.4 \pm 0.22$ ) and less time near the exit $(0.02 \pm 0.06)$ than dogs in the UCC condition (proximity to the box: $0.29 \pm 0.25$; proximity to the exit: $0.09 \pm 0.13$ ).

\section{Vocalization and lip licking}

There were no significant differences between conditions on these measures (all $p$-values $>0.05$ ).

\section{Physiological measures}

\section{Cortisol}

There was no effect of condition [UCC: $1.67 \pm 0.25 \mu \mathrm{g} / \mathrm{dL}$ vs US: $1.37 \pm 0.31 \mu \mathrm{g} / \mathrm{dL}, F(1,42)=0.59, p=0.45]$, time $[F(1,7)=0.16, p=0.70]$, or the condition $\mathrm{x}$ time interaction $[F(1,7)=1.13, p=0.32]$ on cortisol concentrations. We then compared cortisol concentrations between dogs that did and did not open the door in the US condition to asses if the cortisol level of dogs that failed to end their owners' stress (did not open the door) was higher than that of dogs that had a successful response. For dogs in the US condition, the effect of opening the door $[F(1,15)=0.03, p=0.85]$ or the interaction between door opening and time $[F(2,7)=0.31$, $p=0.74]$ did not affect cortisol concentrations.

\section{Heart rate (HR) measured through the POLAR device}

We found a main effect of phase $[F(2,156)=8.42, p<0.001]$, a significant condition $\mathrm{x}$ trial (within phase) interaction $[F(2,156)=3.65, p=0.028]$, and a tendency to reach significance in the interaction between condition and Pre-Post phase $[F(2,156)=2.84, p=0.06]$. No other effects were significant.

Post-hoc comparisons showed that, in the experimental phase (i.e., EXP: taking the mean HR of the three trials), the HR increased compared to the baseline $[t(156)=3.52$, $p=0.001]$, and was higher than after the test $[t(156)=3.72$, $p<0.01]$. Also, the HR was higher after the experiment than at baseline $[t(156)=3.69, p=0.001]$. A closer look at these results reveal that some of the mentioned differences were found only in the US condition (BL vs. EXP: $p=0.001$, EXP vs. POST: $p=0.02$, BL vs. POST: $p=0.38$ ). For the UCC condition, the condition $\mathrm{x}$ trial (within EXP phase) interaction showed that HR decreased across trials. In fact, in the UCC condition, dogs had higher HR in Trial 1 than in Trial $2(p=0.02)$ and Trial $3(p=0.001)$. Meanwhile, no difference across trials was found in the US condition $(p>0.05)$.

\section{Heart rate (HR) measured by auscultation}

We found no differences between conditions in the measure taken before the first trial $(t(36)=0.12, p=0.99)$, whereas dogs in the US condition tended to have higher HR than those in the UCC condition for the measure taken after the last trial $(t(37)=-1.79, p=0.07)$. In fact, the HR increased from the time before to the time after the test in the US condition $(t(17)=-2.50, p=0.02)$, but not in the UCC condition $(t(20)=-0.12, p=0.90)$.

In sum, we observed that dogs in the US condition were more likely to open the door than dogs in the UCC condition. This difference suggests that the motivation to open the door cannot be fully explained by dogs being obedient to their owners' call, given that in Experiment 2, the conditions were yoked in terms of the frequency of calls. In addition, the difference between conditions in the number of door openings also suggests that owners' pretended affective state could have affected dogs' rescue behavior. According to the emotional contagion hypothesis, dogs in the US condition could have been aroused by their owners' shouting and gesturing, making them more likely to open the door than dogs in the UCC condition where owners acted calmly. The evidence of stress differences between conditions is suggestive but, nonetheless, inconclusive. On one hand, we did 
not find differences between conditions in stressed-related behaviors or in cortisol concentrations. On the other hand, we observed differences in HR measures between conditions consistent with the possibility that dogs in the US condition were more aroused by the experimental protocol than those in the UCC condition. These HR results agree with those of Sanford et al. (2018). In conclusion, emotional contagion could be the mechanism involved in dogs' rescue behavior in the present protocol, but the evidence supporting this interpretation is inconclusive.

\section{General discussion}

In the present work, we tested dogs in an experimental paradigm that has been used in other species to study rescue behavior (Bartal et al. 2011; Nowbahari et al. 2009), and we assessed different mechanistic hypotheses of subjects' performance in the task. We set out to investigate if dogs would attempt to open the door of a wooden box, in which their owners pretended to be trapped and were showing signs of distress (screaming, asking for help, hitting the box, and calling the dog).

We found that dogs opened the door more often when their owners acted as if stressed than when they acted calmly. Military dogs trained in search and rescue tasks rescued their stressed trainers a comparable number of times as pet dogs, but with lower latencies. This is the first time that pro-social behavior is documented in dogs using a rescue paradigm. Our findings contrast with the results reported by Sanford et al. (2018) who tested therapy and pet dogs in a similar rescue protocol and did not find significant differences in the proportion of door openings between stressed and calm owner conditions. In the present Experiment 1, unlike in Sanford et al. (2018), trained dogs were faster than untrained dogs in opening the door, and showed decreased latencies across trials. This may suggest that, once trained, dogs became more efficient in responding successfully. Indeed, previous research has shown that training can improve dogs' coping with stressful situations (Diverio et al. 2017; Scandurra et al. 2016), which could have been crucial for dogs to show appropriate responses in the present protocol. The fact that present search and rescue dogs were faster at solving the task but therapy dogs were not (Sanford et al. 2018) suggests that not any kind of training favors the same coping and behavioral strategies. Importantly, in our experiment, trained dogs had not received training in the specific task tested or the apparatus used; however, it could be argued that their everyday activities were more similar to the tested task than those of the therapy dogs tested by Sanford et al. (2018). Possibly, search and rescue dogs in the present Experiment 1 were able to generalize to the experimental situation previously learned copying strategies.
Additionally, overall we found a reduction in the latency to open the door across trials. This could be due to dogs learning that opening the door was rewarding since liberated owners verbally praised their pets. However, opening latencies in stressed and calm conditions were significantly different only for military dogs, suggesting that untrained dogs in the stressed owner condition might have not learned this contingency.

In terms of the mechanisms underlying door openings, in principle, this behavior could have been motivated by the desire to re-establish social contact with the owner. Some previous findings put this explanation at the forefront (Silberberg et al. 2013). First, dogs usually show signs of anxiety when separated from their human attachment figure (Prato-Previde et al. 2003; Topál et al. 1998), and second and consequently, dogs have a pervasive motivation to seek human contact (especially, with their owners) even after brief periods of separation (Parthasarathy and CrowellDavis 2006; Prato-Previde et al. 2003; Rehn et al. 2014). Indeed, the social reinforcement hypothesis obtained some support in a rescue paradigm with rats similar to that used here (Silberberg et al. 2013). In the present experiments, we controlled for this social-contact interpretation by having the calm condition in which the owner was also trapped in the box, that is, was separated from the dog in an identical manner to that of the stressed condition. Therefore, a motivation to seek social contact cannot explain the significant differences in door openings (proportion of dogs and frequencies) between the stressed and calm owner conditions in both Experiments 1 and 2.

In the calm condition in Experiment 1, owners were instructed not to call their dogs or make any verbalization at all. In contrast, owners in the stressed condition were asked to spontaneously ask for help to their pet, which typically involved calling the dog by its name. This procedural difference between conditions was purposely designed, but at the same time, introduced an interpretation issue. On the one hand, from a methodological standpoint, several reasons led us to ask owners to call dogs by their names in stressed owner conditions, but not in the calm owner condition of Experiment 1. We wanted the representation of the owners' distress to be as realistic and spontaneous as possible and preliminary trials had shown that most owners could not avoid calling their dogs while pretending to be trapped, even when we explicitly told them not to do so. In this sense, we understood that, to be spontaneous, owners' calling of dogs needed to involve calling their names. Second, given that this is one of the first studies using this rescue protocol with dogs, we wanted to try the values of the independent variable at their greater contrast. With this goal in mind, we chose to avoid any verbal cue in the calm owner condition. This approach is indeed in line with previous studies with this protocol in other species. For instance, Bartal 
et al. (2011) used a rat shaped toy (Bartal et al. 2011), and Nowbahari et al. (2009) used a chilled motionless ant in their control conditions. Those controls as well as ours involved conditions without any kind of auditory stimuli. On the other hand, this procedural difference left open the possibility that differences in door openings between stressed and calm conditions were the result of the mere obedience of dogs to their owners' call in the stressed condition. In Experiment 2, we controlled for this by instructing owners to calmly call their dogs by their names with a similar frequency to that of the stressed owner condition. Dogs in this calm call condition opened the door significantly less frequently than in the stressed condition which allows us to confidently discard the obedience explanation. Besides, dogs in the calm call condition spent less time interacting or in proximity to the box than dogs in the stress condition. As evidenced in the behavioral analysis, dogs in the calm call condition were mostly uninterested in the box and, importantly, did not respond to the owners' calls, suggesting that the owners' calls were not a strong attention-calling stimulus. The significant difference in the frequency of door openings between calm call and stressed owner conditions suggests that dogs have at least some ability to discriminate human emotional states and act accordingly. Nevertheless, given that in the stressed owner condition we used a compound stimulus (the owner emitted verbal and gestural cues altogether), future studies will be needed to isolate the necessary and sufficient signal elements that trigger dogs' rescue behavior in the present protocol.

Another possible mechanistic explanation of increased door openings in stress relative to calm conditions could be related to dogs' emotional contagion from their owners. In Experiment 2, we took behavioral and physiological measures to test whether dogs' rescue responses could have been driven by a process of emotional contagion, which involves experiencing similar affective states as those observed in another individual. In contrast with the notion of empathic concern (Bartal et al. 2011), emotional contagion does not require individual's recognition of others' internal states (Hatfieldet al. 1994; Panksepp and Panksepp 2013). Interestingly, previous research indicates that dogs can show emotional contagion from their owners (Sümegi et al. 2014; Yong and Ruffman 2014). However, it is important to remark that the idea that dogs could have been driven to open the door by some kind of affective contagion does not necessarily imply that dogs had the goal of relieving their owners' negative affective state. For instance, it could have been the case that dogs got stressed by the situation and tried to find relief by approaching their owner, which led them to open the door. Previous investigations indeed show that dogs may orient towards their owners in situations of uncertainty (D’Aniello et al. 2015; Marshall-Pescini et al. 2013; Scandurra et al. 2015). This interpretation is supported by the fact that dogs' heart rate increased during testing compared to baseline only in the stressed owner group. Also, these results are in line with Sanford et al. (2018), who found higher variation in heart rate responses and higher scores in an anxiety score for dogs in the stressed owner condition than for those in the calm condition.

Despite the mentioned evidence for the emotional contagion hypothesis, results of variations in cortisol levels should make us cautious, because we did not find differences between calm and stressed owner conditions in this variable. Nonetheless, this lack of difference in cortisol levels could be due to external factors that could have increased the noise in the data, thus making difficult to detect a condition effect. The experiment in which we measured the physiological variables was done in an unfamiliar place for subjects (dogs had to be taken to the testing facilities by their owners). In addition, dogs met unfamiliar people there (i.e., the experimenters) which could be an additional stressful factor. All these lead us to consider the possibility of a ceiling effect of the cortisol response caused by extraneous stress. This interpretation is consistent with previous studies showing that dogs' saliva cortisol increased after travelling by car (Bergeron et al. 2002).With this stressful background, it is possible that the stress induced by the stressed owner condition was not sufficient to raise cortisol levels from already high values. Moreover, heart rate differences between stressed and calm conditions could be interpreted as reinforcing the notion that the different ways of responding to stress depend on the different stressors (Moberg 2000; Pacák et al 1998). A clear example of these comes from the work by Beerda et al. (1998) who observed that dogs subjected to different stressors always displayed a clear heart rate response, whereas not all the stressors generated an increase in saliva cortisol. Given the few studies on dogs' stress responses on rescue paradigms, more research would be needed to validate the different physiological responses that dogs manifest in this type of situation.

Last, a cognitively more complex explanation of dogs' rescue behavior involves goal-directedness, namely the possibility that dogs opened the door with the intention to aid the person and end his/her stress. Indeed, this explanation is considered by some authors as a definitive feature of rescue behavior (Nowbahari et al. 2010). It would be interesting to tackle this possibility in future studies by, for example, changing the contingency between the dogs' rescue response and its consequence. For instance, the owner might continue showing signs of stress even after being rescued. If dogs show signs of extinguishing their rescuing behavior in repeated trials of this modified-contingency condition, it would be suggestive of their behavior being goal-directed.

To conclude, present findings indicate that dogs were sensitive to their owners' affective states (stressed vs. calm), and conditionally acted on that information in a rescue paradigm in which they could release their owners from entrapment. 
To our best knowledge, this is the first time that dogs' rescue behavior has been shown in an experimentally controlled protocol. Furthermore, dogs with search and rescue training solved the task faster than untrained dogs. This suggests that learning generalization may play a relevant role in the expression of dogs' pro-sociality towards people (D' Aniello et al. 2015; Scandurra et al. 2015). In terms of the emotional contagion hypothesis, the higher heart rate in the stressed owner condition relative to the calm owner condition presents us with some positive evidence. However, lack of condition differences in cortisol levels and stress behaviors suggests that further studies are needed to be more definitive about the role of this emotional mechanism in dogs' rescue responses. Finally, as in Sanford et al. (2018), approximately half of present dogs opened the box in stressed owner conditions. This makes evident that dogs' reactions to their owners' stress were very diverse. The study of whether and how stable individual differences underlie dogs' pro-sociality, and rescue behaviors in particular, could be an important avenue for future research.

Acknowledgements This work was financially supported by Consejo Nacional de Investigaciones Científicas y Técnicas: PIP 11220130100182, Agencia Nacional de Promoción Científica y Tecnológica: PICT 2014 N0883 to MB and Universidad Nacional del Sur: PGI 24/B243 INBIOSUR to EBC. We would like to express our special gratitude to all the owners who kindly participated in these experiments. We also thank Hugo Carballo, who helped in the building of the apparatus, to the Captain Martin Carbone and the Batallón "Perros de Guerra" who received us at the Puerto Belgrano's military installations, and to Gabriel Granada who helped us finding the facilities to run the tests. Finally we would like to thank the Editor and three anonymous reviewers for their valuable comments which helped to improve the article.

\section{References}

Albuquerque N, Guo K, Wilkinson A, Otta E, Mills D (2016) Dogs recognize dog and human emotions. Biol Lett. https://doi. org/10.1098/rsbl.2015.0883

Barber A, Muller E, Randi D, Muller C, Huber L (2017) Heart rate changes in pet and lab dogs as response to human Facial expressions. ARC J Anim Vet Sci. https://doi.org/10.20431/24552518.0302005

Barrera G, Mustaca A, Bentosela M (2011) Communication between domestic dogs and humans: effects of shelter housing upon the gaze to the human. Anim Cog 14:727-734

Bartal IBA, Decety J, Mason P (2011) Empathy and pro-social behavior in rats. Science 334:1427-1430. https://doi.org/10.1126/scien ce. 1210789

Beerda B, Schilder MB, van Hooff JA, de Vries HW (1997) Manifestations of chronic and acute stress in dogs. Appl Anim Behav Sci 52(3-4):307-319

Beerda B, Schilder MB, van Hooff JA, de Vries HW, Mol JA (1998) Behavioural, saliva cortisol and heart rate responses to different types of stimuli in dogs. Appl Anim Behav Sci 58(3-4):365-381

Bergeron R, Scott SL, Émond JP, Mercier F, Cook NJ, Schaefer AI (2002) Physiology and behavior of dogs during air transport. Can J Vet Res 66(3):211-216
Bräuer J, Schönefeld K, Call J (2013) When do dogs help humans? Appl Anim Behav Sci 148(138):149. https://doi.org/10.1016/j. applanim.2013.07.009

Brosnan SF (2013) Justice- and fairness-related behaviors in nonhuman primates. P Natl Aacad Sci 110:10416-10423. https://doi. org/10.1073/pnas.1301194110

Bshary R, Raihani NJ (2017) Helping in humans and other animals: a fruitful interdisciplinary dialogue. P R Soc B 284:20170929. https ://doi.org/10.1098/rspb.2017.0929

Carballo F, Freidin E, Putrino N, Shimabukuro C, Casanave E, Bentosela M (2015) Dog's discrimination of human selfish and generous attitudes: the role of individual recognition, experience, and experimenters' sex. PLoS ONE 10:e0116314. https://doi. org/10.1371/journal.pone.0116314

Carballo F, Freidin E, Casanave EB, Bentosela M (2017) Dogs' recognition of human selfish and generous attitudes requires little but critical experience with people. PLoS ONE 12:e0185696. https ://doi.org/10.1371/journal.pone.0185696

Carter GG, Wilkinson GS (2013) Food sharing in vampire bats: reciprocal help predicts donations more than relatedness or harassment. P R Soc B280:20122573-20122573. https://doi.org/10.1098/ rspb.2012.2573

Custance D, Mayer J (2012) Emphatic-like responding by domestic dogs (Canis familiaris) to distress in humans: an exploratory study. Anim Cog 15:851-859. https://doi.org/10.1007/s1007 1-012-0510-1

D’Aniello B, Scandurra A, Prato-Previde E, Valsecchi P (2015) Gazing toward humans: a study on water rescue dogs using the impossible task paradigm. Behav Processes 110:68-73. https://doi. org/10.1016/j.beproc.2014.09.022

Damián JP, Bengoa L, Pessina P, Martínez S, Fumagalli F (2018) Serial collection method of dog saliva: Effects of different chemical stimulants on behaviour, volume and saliva composition. Open Vet J 8(3):229-235. https://doi.org/10.4314/ovj.v8i3.1

D'Aniello B, Alterisio A, Scandurra A, Petremolo E, Iommelli MR, Aria M (2017) What's the point? Golden and Labrador retrievers living in kennels do not understand human pointing gestures. Anim Cogn 20:777-787. https://doi.org/10.1007/s 1007 1-017-1098-2

D’Aniello B, Semin GR, Alterisio A, Aria M, Scandurra A (2018) Interspecies transmission of emotional information via chemosignals: from humans to dogs (Canis lupus familiaris). Anim Cogn 21(1):67-78

Diverio S, Menchetti L, Riggio G, Azzari C, Iaboni M, Zasso R, Di Mari W, Santoro M (2017) Dogs' coping styles and dog-handler relationships influence avalanche search team performance. Appl Anim Behav Sci 191:67-77. https://doi.org/10.1016/j.appla nim.2017.02.005

Fallani G, Prato Previde E, Valsecchi P (2007) Behavioral and physiological responses of guide dogs to a situation of emotional distress. Physiol Behav 90:648-655. https://doi.org/10.1016/j.physb eh.2006.12.001. https://doi.org/10.1007/s10071-017-1139-x

Freidin E, Putrino N, D'Orazio M, Bentosela M (2013) Dogs' eavesdropping from people's reactions in third party interactions. PLoS ONE 8:e79198. https://doi.org/10.1371/journal.pone.0079198

Freidin E, Carballo F, Bentosela M (2017) Direct reciprocity in animals: the roles of bonding and affective processes. Int J Psychol 52:163-170. https://doi.org/10.1002/ijop.12215

Galibert F, Quignon P, Hitte C, André C (2011) Toward understanding dog evolutionary and domestication history. CR Biol 334(3):190196. https://doi.org/10.1016/j.crvi.2010.12.011

Gfrerer N, Taborsky M (2017) Working dogs cooperate among one another by generalized reciprocity. Nature Sci Rep 7:43-67. https ://doi.org/10.1038/srep43867

Hare B, Tomasello M (2005) Human-like social skills in dogs? Trends Cogn Sci 9:439-444. https://doi.org/10.1016/j.tics.2005.07.003 
Hatfield E, Cacioppo JT, Rapson RL (1994) Emotional contagion: Studies in emotion and social interaction. Editions de la Maison des sciences de l'homme.

Huber A, Barber AL, Faragó T, Müller CA, Huber L (2017) Investigating emotional contagion in dogs (Canis familiaris) to emotional sounds of humans and conspecifics. Anim Cogn 20(4):703-715. https://doi.org/10.1007/s10071-017-1092-8

Jezierski T, Adamkiewicz E, Walczak M, Sobczyńska M, GóreckaBruzda A, Ensminger J, Papet E (2014) Efficacy of drug detection by fully-trained police dogs varies by breed, training level, type of drug and search environment. Forensic Sci Int 237:112-118. https://doi.org/10.1016/j.forsciint.2014.01.013

Kaminski J, Neumann M, Bräuer J, Call J, Tomasello M (2011) Dogs, Canis Faniliaris, Communicate with humans to request but not to inform. Anim Behav 82:651-658

Kaminski J, Nitzschner M (2013) Do dogs get the point? A review of dog-human communication ability Learn Motiv 44294302 10.1016/j.lmot.2013.05.001.

King T, Hemsworth PH, Coleman GJ (2003) Fear of novel and startling stimuli in domestic dogs. Appl Anim Behavr Sci 82(1):45-64. https://doi.org/10.1016/S0168-1591(03)00040-6

Kundey S, De Los RA, Arbuthnot J, Allen R, Cochum A, Molina S, Royer E (2010a) Domesticated dogs' (Canis familiris) response to dishonest human points. Int J Comp Psychol 23:201-215

Kundey S, De Los Reyes A, Royer E, Molina S, Monnier B, German R, Coshun A (2010b) Reputation-like inference in domestic dogs (Canis familiris). Anim Cogn 14:291-302. https://doi. org/10.1007/s10071-010-0362-5

Macpherson K, Roberts WA (2006) Do dogs (Canis familiris) seek help in an emergency? J Comp Psychol 120:113-119. https://doi. org/10.1037/0735-7036.120.2.113

Marshall-Pescini S, Passalacqua C, Barnard S, Valsecchi P, Prato-Previde $\mathrm{E}$ (2009) Agility and search and rescue training differently affects pet dogs' behaviour in socio-cognitive tasks. Behav Proc 81(3):416-422. https://doi.org/10.1016/j.beproc.2009.03.015

Marshall-Pescini S, Passalacqua C, Ferrario A, Valsecchi P, PratoPrevide E (2011) Social eavesdropping in the domestic dog. Anim Behav 81:1177-1183. https://doi.org/10.1016/j.anbeh av.2011.02.029

Marshall-Pescini S, Colombo E, Passalacqua C, Merola I, Prato-Previde $\mathrm{E}$ (2013) Gaze alternation in dogs and toddlers in an unsolvable task: evidence of an audience effect. Anim Cogn 16:933-943. https://doi.org/10.1007/s10071-013-0627-x

Marshall-Pescini S, Dale R, Quervel-Chaumette M, Range F (2016) Critical issues in experimental studies of prosociality in nonhuman species. Anim Cogn 19:679-705. https://doi.org/10.1007/ s10071-016-0973-6

McGowan RT, Bolte C, Barnett HR, Perez-Camargo G, Martin F (2018) Can you spare $15 \mathrm{~min}$ ? The measurable positive impact of a 15-min petting session on shelter dog well-being. Appl Anim Behav Sci 203:42-54. https://doi.org/10.1016/j.appla nim.2018.02.011

Merola I, Prato-Previde E, Lazzaroni M, Marshall-Pescini S (2013) Dogs' comprehension of referential emotional expressions: familiar people and familiar emotions are easier. Anim Cogn 17:373385. https://doi.org/10.1007/s10071-013-0668-1

Miklósi Á, Topál J (2013) What does it take to become "best friends"? Evolutionary changes in canine social competence. Trends Cogn Sci 17:287-294. https://doi.org/10.1016/j.tics.2013.04.005

Moberg GP (2000) Biological response to stress: implications for animal welfare. In: Moberg GP, Mench JA (eds) The biology of animal stress: basic principles and implications for animal welfare. CABI Publishing, Wallingford, Oxon, UK, pp 1-22

Müller CA, Schmitt K, Barber ALA, Huber L (2015) Dogs can discriminate emotional expressions of human faces. Curr Biol 25:601-605. https://doi.org/10.1016/j.cub.2014.12.055
Nagasawa M, Mitsui S, En S, Ohtani N, Ohta M, Sakuma Y, Onaka T, Mogi K, Kikusui T (2015) Oxytocin-gaze positive loop and the coevolution of human-dog bonds. Science 348(6232):333-336. https://doi.org/10.1126/science.1261022

Nitzschner M, Melis AP, Kaminski J, Tomasello M (2012) Dogs (Canis familiris) Evaluate humans on the basis of direct experiences only. PLoS ONE 7:e46880. https://doi.org/10.1371/journal.pone.00468 80

Nowak MA (2006) Five rules for the evolution of cooperation. Science 314:1560-1563. https://doi.org/10.1126/science.1133755

Nowbahari E, Hollis K (2010) Distinguishing between recue, cooperation and other forms of altruistic behavior. Communicative and Integrative Biology 3(2):77-79

Nowbahari E, Scohier A, Durand J-L, Hollis KL (2009) Ants, cataglyphis cursor, use precisely directed rescue behavior to free entrapped relatives. PLoS ONE 4:e6573. https://doi.org/10.1371/ journal.pone.0006573

Pacák K, Palkovits M, Yadid G, Kvetnansky R, Kopin IJ, Goldstein DS (1998) Heterogeneous neurochemical responses to different stressors: a test of Selye's doctrine of nonspecificity. Am J Physiol Regul Integr Comp Physiol 275:1247-1255

Palestrini C, Previde EP, Spiezio C, Verga M (2005) Heart rate and behavioural responses of dogs in the Ainsworth's Strange Situation: A pilot study. Appl Anim Behav Sci 94:75-88. https://doi. org/10.1016/j.applanim.2005.02.005

Panksepp J, Panksepp JB (2013) Toward a cross-species understanding of empathy. Trends Neurosci 36:489-496. https://doi. org/10.1016/j.tins.2013.04.009

Parthasarathy V, Crowell-Davis SL (2006) Relationship between attachment to owners and separation anxiety in pet dogs (Canis lupus familiris). J Vet Behav 1:109-120. https://doi.org/10.1016/j. jveb.2006.09.005

Plotnik JM, Lair R, Suphachoksahakun W, de Waal FBM (2011) Elephants know when they need a helping trunk in a cooperative task. P Natl Aacad Sci 108:5116-5121. https://doi.org/10.1073/ pnas. 1101765108

Prato-Previde E, Custance DM, Spiezio C, Sabatini F (2003) Is the dog-human relationship an attachment bond? An observational study using Ainsworth's strange situation. Behaviour 140:225254. https://doi.org/10.1163/156853903321671514

Quervel-Chaumette M, Dale R, Marshall-Pescini S, Range F (2015) Familiarity affects other-regarding preferences in pet dogs. Sci Rep Uk 5:18102. https://doi.org/10.1038/srep18102

Quervel-Chaumette M, Mainix G, Range F, Marshall-Pescini S (2016) Dogs Do Not Show Pro-social Preferences towards Humans. Front Psychol 7:1416. https://doi.org/10.3389/fpsyg.2016.01416

Rehn T, Handlin L, Uvnäs-Moberg K, Keeling LJ (2014) Dogs' endocrine and behavioural responses at reunion are affected by how the human initiates contact. Physiol Behav 124:45-53. https://doi. org/10.1016/j.physbeh.2013.10.009

Sanford EM, Burt ER, Meyers-Manor JE (2018) Timmy's in the well: Empathy and prosocial helping in dogs. Learning \& behavior 46(4):374-386. https://doi.org/10.3758/s13420-018-0332-3

Scandurra A, Prato-Previde E, Valsecchi P, Aria M, D'Aniello B (2015) Guide dogs as a model for investigating the effect of life experience and training on gazing behavior. Anim Cogn 18:937-944. https://doi.org/10.1007/s10071-015-0864-2

Scandurra A, Alterisio A, D'Aniello B (2016) Behavioural effects of training on water rescue dogs in the Strange Situation Test. Appl Anim Behavr Sci 174:121-127

Schwab C, Huber L (2006) Obey or not obey? Dogs (Canis familiaris) behave differently in response to attentional states of their owners. J Comp Psychol 120:69-175. https://doi. org/10.1037/0735-7036.120.3.169

Silberberg A, Allouch C, Sandfort S, Kearns D, Karpel H, Slotnick B (2013) Desire for social contact, not empathy, may explain 
"rescue" behavior in rats. Anim Cogn 17:609-618. https://doi. org/10.1007/s10071-013-0692-1

Sümegi Z, Oláh K, Topál J (2014) Emotional contagion in dogs as measured by change in cognitive task performance. Appl Anim Behav Sci 160:106-115. https://doi.org/10.1016/j.appla nim.2014.09.001

Szetei V, Miklósi Á, Topál J, Csányi V (2003) When dogs seem to lose their nose: an investigation on the use of visual and olfactory cues in communicative context between dog and owner. Appl Anim Behav Sci 83:141-152. https://doi.org/10.1016/s0168 -1591(03)00114-x

Topál J, Miklósi Á, Csányi V, Dóka A (1998) Attachment Behavior in Dogs (Canis familiris): A New Application of Ainsworth's (1969) Strange Situation Test. J Comp Psychol 112(3):219-229

Udell MA, Dorey N, Wynne CDL (2008) Wolves outperform dogs in following human social cues. Anim Behav 76:1767-1773. https ://doi.org/10.1016/j.anbehav.2008.07.028

Vasconcelos M, Hollis K, Nowbahari E, Kacelnik A (2012) Pro-sociality without empathy. Biol Lett 8:910-912. https://doi.org/10.1098/ rsbl.2012.0554

Vilà C, Savolainen P, Maldonado JE, Amorim IR, Rice JE, Honeycutt RL, Crandall KA, Lundeberg J, Wayne RK (1997) Multiple and ancient origins of the domestic dog. Science 276:1687-1689. https ://doi.org/10.1126/science.276.5319.1687
Walker JK, Dale AR, D'Eath RB, Wemelsfelder F (2016) Qualitative Behaviour Assessment of dogs in the shelter and home environment and relationship with quantitative behaviour assessment and physiological responses. Appl Anim Behav Sci 184:97-108. https ://doi.org/10.1016/j.applanim.2016.08.012

Wallis LJ, Range F, Müller CA, Serisier S, Huber L, Virányi Z (2015) Training for eye contact modulates gaze following in dogs. Anim Behav 106:27-35. https://doi.org/10.1016/j.anbehav.2015.04.020

Warneken F (2015) Precocious Prosociality: Why Do Young Children Help? Child Dev Perspect 9:1-6. https://doi.org/10.1111/ cdep. 12101

Yong MH, Ruffman T (2014) Emotional contagion: Dogs and humans show a similar physiological response to human infant crying. Behav Processes 108:155-165. https://doi.org/10.1016/j.bepro c. 2014.10 .006

Publisher's Note Springer Nature remains neutral with regard to jurisdictional claims in published maps and institutional affiliations. 